\begin{tabular}{llllll} 
O P E R A T I O N S R E S E A R C H A N D D E C I S I O N S \\
\hline No. 1
\end{tabular}

DOI: $10.37190 /$ ord200105

\title{
GOAL PROGRAMMING APPROACH FOR SOLVING HEPTAGONAL FUZZY TRANSPORTATION PROBLEM UNDER BUDGETARY CONSTRAINT
}

\author{
HAMIDEN ABD EL-WAHED KHALIFA* \\ Operations Research Department, Faculty of Graduate Studies for Statistical Research, \\ Cairo University, Giza, Egypt
}

\begin{abstract}
Transportation problem (TP) is a special type of linear programming problem (LPP) where the objective is to minimize the cost of distributing a product from several sources (or origins) to some destinations. This paper addresses a transportation problem in which the costs, supplies, and demands are represented as heptagonal fuzzy numbers. After converting the problem into the corresponding crisp TP using the ranking method, a goal programming (GP) approach is applied for obtaining the optimal solution. The advantage of GP for the decision-maker is easy to explain and implement in real life transportation. The stability set of the first kind corresponding to the optimal solution is determined. A numerical example is given to highlight the solution approach.
\end{abstract}

Keywords: transportation problem, heptagonal fuzzy numbers, ranking method, goal programming, parametric study

\section{Introduction}

Transportation problem (TP) is a special type of linear programming (LP) problem where the objective is to minimize the cost of distributing product from $m$ sources or origins to $n$ distributions, and their capacities are $u_{1}, u_{2}, \ldots, u_{m}$ and $v_{1}, v_{2}, \ldots, v_{n}$, respectively. In addition, there is a penalty $c_{i j}$ associated with transportation a unit of product from source $i$ to destination $j$. This penalty may be cost or delivery time of safety of delivery, etc. A variable $x_{i j}$ represents the unknown quantity to be shipped from source $i$ to destination $j$.

*Present address: Mathematics Department, College of Science and Arts, Al- Badaya, Qassim University, Saudi Arabia, e-mail address: ha.Ahmed@qu.edu.sa

Received 30 June 2019, accepted 18 April 2020 
One of the difficulties which occur in the application of mathematical programming is that the parameters in the problem formulation are not constants but fluctuating and uncertain. Transportation or shipping problem (TP) involves determining the number of goods or items to be transported from many sources to several destinations. TP is a specific case of linear programming (LP) problems and a special algorithm has developed to solve it.

In many scientific areas such as system analysis and operator research, a model has to be set up using the data which is known only approximately. Fuzzy sets theory, introduced by Zadeh [23], makes this possible. Fuzzy numerical data can be represented employing fuzzy subsets of the real line, known as fuzzy numbers. Dubois and Prade [5] extended the use of algebraic operations on real numbers to fuzzy numbers through fuzzification principle. Bellman and Zadeh [2] introduced the concept of fuzzy set theory into the decision-making problem involving uncertainty and imprecision. Tanaka et al. [22] formulate a fuzzy linear programming problem to obtain a reasonable solution under consideration of the ambiguity of parameters. Gani and Abbas [6] proposed a new method for finding an optimal solution for intuitionistic fuzzy transportation problem (FTP) based on a new ranking method. Narayanamoorthy et al. [13] offer a new algorithm, called Russell's method, for solving fuzzy TP. Senthilkumar and Vengataasalam [21] discussed and solved the fuzzy transportation problem with fuzzy quantities in two stages. Jaikumar [7] extends the approach introduced by Ahmed et al. [1] into fuzzified form to TP. Malini and Kennedy [10] studied TP with octagonal fuzzy numbers, using the ranking method. Kaur and Kumar [8] suggest a new method for solving FTPs by assuming that a decision-maker (DM) is uncertain about the cost coefficients, availability, and demand of the product. Melita Vinoliah and Ganesan [12] give a new method for obtaining a good initial basic feasible solution of FTP in which all the costs, supplies, and demands are trapezoidal fuzzy numbers, without converting to classical TPs. Pathade and Ghadle [16] examine FTP with all of the cost, supply, and demand are octagonal fuzzy numbers. Ramesh Kumar and Subramanian [19] solved TP using the Robust ranking technique where demand and supply are represented in the trapezoidal fuzzy numbers. Mathur and Srivastava [11] discussed a new approach using hexagonal fuzzy numbers in TP with a fuzzy environment. Chhibber et al. [4] employed in centre of centroids to convert trapezoidal FTP of type 1 and type 2 both into crisp one, and hence resolved it by north-west corner technique to obtain the primary solution, and optimality it is checked through modified distribution method. Maheswari and Vijaya [9] developed a methodology for finding an initial basic feasible solution of FTP based on the ranking technique of trapezoidal fuzzy numbers using the centroid of incenters. Rathi and Balamohan [18] introduced a new form of non-normal fuzzy number named heptagonal fuzzy number and defined its arithmetic operations.

In his earlier work, Osman [14] introduced the notions of the solvability set, the stability set of the first kind, and the stability set of the second kind, and analyzed these concepts for parametric convex non-linear programming problems. Osman and El- 
-Banna [15] introduced the stability of multiobjective nonlinear programming problems with fuzzy parameters.

In this paper, a goal programming approach is applied for solving the heptagonal fuzzy numbers transportation problem under budgetary constraint, and the stability set of the first kind corresponding to the obtaining solution is determined.

The outlay of the paper was organized as follows: Section 2 presented some preliminary considerations. Section 3 introduced the notation and indices involved in the problem formulation. Section 4 introduced a heptagonal fuzzy numbers transportation problem under budgetary constraint. Section 5 gave a solution procedure for obtaining a fuzzy optimal solution for the problem. Section 6 investigated a numerical example to illustrate the proposed approach. Finally, some concluding remarks have been reported in Section 7.

\section{Preliminaries}

In this section, the basic concepts and results related to fuzzy numbers, and heptagonal fuzzy numbers and some of the arithmetic operations are recalled $[3,17,20]$.

Definition 1. A fuzzy set $\tilde{A}$ defined on the set of real numbers $\mathbb{R}$ is said to be fuzzy numbers if its membership function $\mu_{\tilde{A}}(x): \mathbb{R} \rightarrow[0,1]$ has the following property

- $\mu_{\tilde{A}}(x)$ is an upper semi-continuous membership function,

- $\tilde{A}$ is a convex fuzzy set, i.e., $\mu_{\tilde{A}}(\gamma x+(1-\gamma) y) \geq \min \left\{\mu_{\tilde{A}}(\mathrm{x}), \mu_{\tilde{A}}(y)\right\} ; \forall x, y \in \mathbb{R} ; \forall 0 \leq \gamma \leq 1$,

- $\tilde{A}$ is normal, i.e., $\exists x_{0} \in \mathbb{R}$ for which $\mu_{\tilde{A}}\left(x_{0}\right)=1$,

- $\operatorname{Supp}(\tilde{A})=\left\{x \in \mathbb{R}: \mu_{\tilde{A}}(x)>0\right\}$ is the support of $\tilde{A}$, and the closure $\operatorname{cl}(\operatorname{Supp}(\tilde{A}))$ is a compact set.

Definition 2. The $\alpha$-cut of the fuzzy number $\tilde{A}, \alpha \in[0,1]$ is denoted by $(\tilde{A})_{\alpha}$ and is defined as the ordinary set:

$$
(\tilde{A})_{\alpha}=\left\{\begin{array}{l}
x \in \mathbb{R}: \mu_{\tilde{A}}(x) \geq \alpha, 0 \leq \alpha \leq 1 \\
\operatorname{cl}(\operatorname{Supp}(\tilde{A})), \quad \alpha=0
\end{array}\right.
$$


Definition 3. A fuzzy number $\tilde{H}$ of $\mathbb{R}$ is said to be a heptagonal fuzzy number denoted as $\tilde{P}_{H}=\left(h_{1}, h_{2}, h_{3}, h_{4}, h_{5}, h_{6}, h_{7}\right)$ if its membership function $\mu_{\tilde{P}_{H}}: \mathbb{R} \rightarrow[0,1]$ has the following properties (Fig. 1):

$$
\mu_{\tilde{P}_{H}}(x)=\left\{\begin{array}{l}
0, \text { for } x<h_{1} \\
\frac{1}{2}\left(\frac{x-h_{1}}{h_{2}-h_{1}}\right) \quad \text { for } h_{1} \leq x \leq h_{2} \\
\frac{1}{2} \text { for } h_{2} \leq x \leq h_{3} \\
\frac{1}{2}+\frac{1}{2}\left(\frac{x-h_{3}}{h_{4}-h_{3}}\right) \text { for } h_{3} \leq x \leq h_{4} \\
\frac{1}{2}+\frac{1}{2}\left(\frac{h_{5}-x}{h_{5}-h_{4}}\right) \text { for } h_{4} \leq x \leq h_{5} \\
\frac{1}{2} \text { for } h_{5} \leq x \leq h_{6} \\
\frac{1}{2}\left(\frac{h_{7}-x}{h_{7}-h_{6}}\right) \text { for } h_{6} \leq x \leq h_{7} \\
0 \text { for } x \geq h_{7}
\end{array}\right.
$$

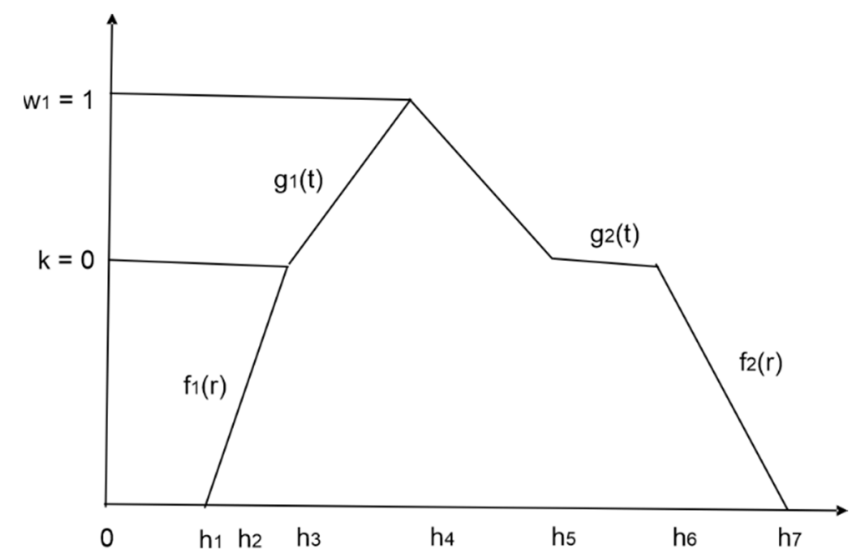

Fig. 1. Heptagonal fuzzy number graphical representation

A heptagonal fuzzy number $\tilde{P}_{H}=\left(h_{1}, h_{2}, h_{3}, h_{4}, h_{5}, h_{6}, h_{7}\right)$ can be represented as in the parametric $\tilde{H}_{p}=\left(f_{1}(r), g_{1}(t), g_{2}(t), f_{2}(r)\right) ; 0 \leq r \leq k ; k \leq t \leq 1$, where $f_{1}(r)$ and $g_{1}(t)$ 
are bounded left continuous non-decreasing functions over $\left[0, w_{1}\right]$ and $\left[k, w_{2}\right]$, respectively, $f_{2}(r)$ and $g_{2}(t)$ are bounded left continuous non-increasing functions over $\left[0, w_{1}\right]$ and $\left[k, w_{2}\right]$, respectively.

Let $\tilde{A}_{H}=\left(a_{1}, a_{2}, a_{3}, a_{4}, a_{5}, a_{6}\right)$ and $\tilde{D}_{H}=\left(b_{1}, b_{2}, b_{3}, b_{4}, b_{5}, b_{6}, b_{7}\right)$ be two heptagonal fuzzy numbers. Then, the arithmetic operations on $\tilde{A}_{H}$ and $\tilde{B}_{H}$ as addition and subtraction can be defined as follows:

Addition:

$$
\tilde{A}_{H} \oplus \tilde{D}_{H}=\left(a_{1}+b_{2}, a_{2}+b_{2}, a_{3}+b_{3}, a_{4}+b_{4}, a_{5}+b_{5}, a_{6}+b_{6}, a_{7}+b_{7}\right)
$$

Subtraction:

$$
\tilde{A}_{H}(-) \tilde{D}_{H}=\left(a_{1}-b_{7}, a_{2}-b_{6}, a_{3}-b_{5}, a_{4}-b_{4}, a_{5}-b_{3}, a_{6}-b_{2}, a_{7}-b_{1}\right)
$$

Definition 4. If $\tilde{P}_{H}=\left(h_{1}, h_{2}, h_{3}, h_{4}, h_{5}, h_{6}, h_{7}\right)$ be a heptagonal fuzzy number, then, the ranking function $R\left(\tilde{P}_{H}\right)$ is defined as:

$$
\begin{aligned}
R\left(\tilde{P}_{H}\right)= & \int_{0}^{1} 0.5\left(a_{h_{\alpha}^{-}}, a_{h_{\alpha}^{+}}\right) d \alpha \\
= & \int_{0}^{1} 0.5\left\{\left(h_{2}-h_{1}\right) \alpha+h_{1}, h_{4}-\left(h_{4}-h_{3}\right) \alpha,\left(h_{6}-h_{5}\right) \alpha+h_{5},\right. \\
& \left.h_{7}-\left(h_{7}-h_{5}\right) \alpha\right\} d \alpha ; \forall \alpha \in[0,1]
\end{aligned}
$$

\section{Problem formulation and solution concepts}

\subsection{Indices and notations}

\section{Indices}

$i \quad$ - source index for all $i=1,2, \ldots, m$

$j \quad-$ destination index for all $j=1,2, \ldots, n$

\section{Notations}

$x_{i j}-$ number of units of the commodity transported from $i$ th source to $j$ th destination

$\tilde{c}_{i j_{H}}-$ heptagonal fuzzy cost of one unit commodity transported from $i$ th source to $j$ th destination 
$x_{i}-$ total availability of the product at the source $i$

$x_{j}-$ total demand of the product at the destination $j$

$\tilde{a}_{i_{H}}-$ quantity of a commodity available at the $i$ th source

$\tilde{b}_{j_{H}}-$ quantity of a commodity demand at the $j$ th destination

$\tilde{B}_{H}-$ budget allotted from transportation

\subsection{Problem formulation}

Consider transportation problem with $m$ sources and $n$ destinations in which all of $\tilde{a}_{i_{H}}, \tilde{b}_{j_{H}}, \tilde{c}_{i_{H}}$ and $\tilde{B}_{H}$ are heptagonal fuzzy numbers. The problem is to find the optimum values of $x_{i j}$ such that:

$$
\begin{aligned}
& \sum_{j=1}^{n} x_{i j} \leq \tilde{a}_{i_{H}}, i=1,2, \ldots, m \\
& \sum_{i=1}^{m} x_{i j} \leq \tilde{b}_{i j} \geq \tilde{b}_{j_{H}}, j=1,2, \ldots, n \\
& \sum_{i=1}^{m} \sum_{j=1}^{n} \tilde{c}_{i j_{H}} x_{i j} \leq \tilde{B}_{H} \\
& x_{i j} \geq 0, i=1,2, \ldots, m, j=1,2, \ldots, n
\end{aligned}
$$

Definition 5. A transportation problem with one or more fuzzy parameters is defined as a fuzzy transportation problem (FTP). As on definition 4, problem (1) can be converted into its corresponding crisp as

$$
\begin{aligned}
& \sum_{j=1}^{n} x_{i j} \leq R\left(\tilde{a}_{i_{H}}\right), i=1,2, \ldots, m \\
& \sum_{i=1}^{m} x_{i j} \geq R\left(\tilde{b}_{j_{H}}\right), j=1,2, \ldots, n \\
& x_{i j} \geq 0, i=1,2, \ldots, m, j=1,2, \ldots, n
\end{aligned}
$$

The goal-programming version for problem (2) is as follows:

$$
\min V(d)=\sum_{j=1}^{n} d_{j}^{-}
$$

subject to 


$$
\begin{aligned}
& \sum_{j=1}^{n} x_{i j} \leq R\left(\tilde{a}_{i_{H}}\right), i=1,2, \ldots, m \\
& \sum_{i=1}^{m} x_{i j}-d_{j}^{+}+d_{j}^{-}=R\left(\tilde{b}_{j_{H}}\right), j=1,2, \ldots, n \\
& \sum_{i=1}^{m} \sum_{j=1}^{n} R\left(\tilde{c}_{i j_{H}}\right) x_{i j} \leq R\left(\tilde{B}_{H}\right) \\
& x_{i j}, d_{j}^{+}, d_{j}^{-} \geq 0, i=1,2, \ldots, m, j=1,2, \ldots, n \\
& d_{j}^{+}, d_{j}^{-}=0, j=1,2, \ldots, n
\end{aligned}
$$

\section{Solution procedure}

In this section, a solution procedure for solving the problem (1) is developed in the following steps:

Step 1. Construct the fuzzy transportation problem (1), and then convert it into a balanced one if it is not.

Step 2. Convert the problem (1) into the corresponding crisp transportation problem (2) based on the ranking function as in definition 4 .

Step 3. Apply fuzzy goal programming approach for the problem (2).

Step 4. Use GAMS software to solve the goal-programming version (3).

Step 5. Determine $S\left(x^{*}, b^{*}\right)$ by applying the following conditions:

$$
\begin{aligned}
& \gamma_{j}\left(b_{j}^{*}-e_{2 j}\right)=0, j=1,2, \ldots, n ; \\
& \mu_{j}\left(e_{1 j}-b_{j}^{*}\right)=0, j=1,2, \ldots, n ; \\
& \gamma_{j}, \mu_{j} \geq 0, b_{j} \in\left[e_{1 j}, e_{2 j}\right], j=1,2, \ldots, n
\end{aligned}
$$

Considering the following cases:

Case 1. $\gamma_{j}>0, j \in J_{1} \subset\{1,2, \ldots, n\}, \gamma_{j}=0, j \notin J_{1}, \mu_{j}>0, j \notin J_{2} \subset\{1,2, \ldots, n\}$, $\mu_{j}=0, j \notin J_{2}$.

Let $N$ be the set of all proper subsets of $\{1,2, \ldots, n\}$. Then,

$$
S_{J_{1}, J_{2}}\left(x^{*}, b^{*}\right)=\left\{\begin{array}{l}
\left(e_{1}, e_{2}\right) \in \mathbb{R}^{2 n}: e_{2 j}=b_{j}^{*}, j \in J_{1}, e_{2 j} \geq b_{j}^{*}, j \notin J_{1} \\
e_{2 j}=b_{j}^{*}, j \in J_{2}, e_{i j} \leq b_{j}^{*}, j \notin J_{2}
\end{array}\right\}
$$


Hence,

$$
S_{1}\left(x^{*}, b^{*}\right)=\bigcup_{J_{1}, J_{2}} \mathrm{~S}_{J_{1}, J_{2}}\left(x^{*}, b^{*}\right)
$$

Case 2. $\gamma_{j}, \mu_{j}=0$

Then,

$$
S_{2}\left(x^{*}, b^{*}\right)=\left\{\left(e_{1}, e_{2}\right) \in \mathbb{R}^{2 n}: e_{2 j} \geq b_{j}^{*}, j=1,2, \ldots, n, e_{1 j} \leq b_{j}^{*}, j=1,2, \ldots, n\right\}
$$

Case 3. $\gamma_{j}, \mu_{j}>0$

Then,

$$
S_{3}\left(x^{*}, b^{*}\right)=\left\{\left(e_{1}, e_{2}\right) \in \mathbb{R}^{2 n}: e_{2 j}=b_{j}^{*}, j=1,2, \ldots, n ; e_{1 j}=b_{j}^{*}, j=1,2, \ldots, n\right\}
$$

Thus,

$$
S\left(x^{*}, b^{*}\right)=\bigcup_{q=1}^{3} S_{q}\left(x^{*}, b^{*}\right)
$$

\section{Numerical example}

Consider the FTP with three sources $S_{1}, S_{2}, S_{3}$ and three destinations $D_{1}, D_{2}, D_{3}$, then the fuzzy transportation cost $\tilde{c}_{i j_{H}}$ is

$$
\tilde{c}_{i j_{H}}=\left[\begin{array}{ccc}
(3,6,2,1,5,0,4) & (2,3,1,4,3,6,5) & (2,4,3,1,6,5,2) \\
(2,7,7,6,3,2,1) & (1,3,5,7,9,11,13) & (0,1,2,4,6,0,5) \\
(3,6,3,2,1,8,7) & (3,4,3,2,1,1,0) & (2,4,6,8,10,12,14)
\end{array}\right]
$$

The fuzzy availabilities of the supply are

$$
(2,2,1,2,1,1,0),(3,2,1,4,5,0,1),(2,4,3,1,6,5,2)
$$

and the fuzzy availabilities of the demand are

$$
(1,3,5,7,9,11,13),(3,6,2,1,5,0,4),(2,4,3,1,6,5,2)
$$


Table 1. Heptagonal fuzzy transportation problem

\begin{tabular}{|c|c|c|c|c|}
\hline \multirow{2}{*}{ Origin } & \multicolumn{3}{|c|}{ Destination } & \multirow{2}{*}{ Supply } \\
\cline { 2 - 4 } & $D_{1}$ & $D_{2}$ & $D_{3}$ & \\
\hline$S_{1}$ & $(3,6,2,1,5,0,4)$ & $(2,3,1,4,3,6,5)$ & $(2,4,3,1,6,5,2)$ & $(2,2,1,2,1,1,0)$ \\
\hline$S_{2}$ & $(2,7,7,6,3,2,1)$ & $(1,3,5,7,9,11,13)$ & $(0,1,2,4,6,0,5)$ & $(3,2,1,4,5,0,1)$ \\
\hline$S_{3}$ & $(3,6,3,2,1,8,7)$ & $(3,4,3,2,1,1,0)$ & $(2,4,6,8,10,12,14)$ & $(2,4,3,1,6,5,2)$ \\
\hline Demand & $(1,3,5,7,9,11,13)$ & $(3,6,2,1,5,0,4)$ & $(2,4,3,1,6,5,2)$ & \\
\hline
\end{tabular}

Step 2. Convert the problem (1) into the corresponding crisp transportation problem (2) based on the ranking function as in definition 4 .

$$
\tilde{c}_{i j_{H}}=\left[\begin{array}{ccc}
6 & 6.75 & 7.25 \\
8.25 & 14.5 & 7 \\
7.75 & 16.5 & 10.5
\end{array}\right]
$$

Table 2. Crisp transportation problem

\begin{tabular}{|c|c|c|c|c|}
\hline \multirow{2}{*}{ Origin } & \multicolumn{3}{|c|}{ Destination } & \multirow{2}{*}{ Supply } \\
\cline { 2 - 4 } & $D_{1}$ & $D_{2}$ & $D_{3}$ & \\
\hline$S_{1}$ & 6 & 6.75 & 7.25 & 2.5 \\
\hline$S_{2}$ & 8.25 & 14.5 & 7 & 10.5 \\
\hline$S_{3}$ & 7.75 & 16.5 & 10.5 & 14.5 \\
\hline Demand & 14.5 & 6 & 7 & \\
\hline
\end{tabular}

Step 3. The fuzzy goal model for the transportation problem is

$$
\min V(d)=\sum_{j=1}^{3} d_{j}^{-}
$$

subject to

$$
\begin{aligned}
& x_{11}+x_{12}+x_{13} \leq 2.5 \\
& x_{21}+x_{22}+x_{23} \leq 10.5 \\
& x_{31}+x_{32}+x_{33} \leq 14.5 \\
& x_{11}+x_{21}+x_{31}-d_{1}^{+}+d_{1}^{-}=14.5 \\
& x_{12}+x_{22}+x_{32}-d_{2}^{+}+d_{2}^{-}=6 \\
& x_{13}+x_{23}+x_{33}-d_{3}^{+}+d_{3}^{-}=7
\end{aligned}
$$




$$
\begin{aligned}
& \left(\begin{array}{l}
6 x_{11}+6.75 x_{12}+7.25 x_{13} \\
8.25 x_{21}+14.5 x_{22}+7 x_{23} \\
+7.75 x_{31}+16.5 x_{32}+10.5 x_{33}
\end{array}\right) \leq 280.375 \\
& x_{i j}, d_{j}^{+}, d_{j}^{-} \geq, i=j=1,2,3 \\
& d_{j}^{-}, d_{j}^{+}=0, j=1,2,3
\end{aligned}
$$

Since all goals are equally priority, we solve the problem using the software GAMS. The solution details illustrated as in the following table:

Table 3. Solution of the problem (4)

\begin{tabular}{|c|c|c|c|c|}
\hline \multirow{2}{*}{ Origin } & \multicolumn{3}{|c|}{ Destination } & \multirow{2}{*}{ Supply } \\
\cline { 2 - 5 } & $D_{1}$ & $D_{2}$ & $D_{3}$ & \\
\hline$S_{1}$ & 0 & 0 & 2.5 & 2.5 \\
\hline$S_{2}$ & 0 & 6 & 4.50 & 10.50 \\
\hline$S_{3}$ & 14.50 & 0 & 0 & 14.50 \\
\hline Existing demand & 14.50 & 6 & 7 & 27.50 \\
\hline Fulfilled demand & 14.50 & 6 & 7 & 27.50 \\
\hline Deficit & 0 & 0 & 0 & 0 \\
\hline
\end{tabular}

It is clear that there is no deficit in the demands; this is because the goal programming is based on the minimizing sum principle of the deviations from the goal values, and the achievement function value as $V(d)=0$.

To reduce the computational effort through the determination $S(2.5,6.4,4.5,14.5,6.7)$, we confirm the left-hand side of the following intervals $0 \leq b_{1} \leq 14.4,0 \leq b_{2} \leq 6,2.5 \leq b_{3} \leq 7$.

Hence, $S(2.5,6,4.5,14.5,14.5,6,7)$ is determined by the following conditions:

$$
\begin{aligned}
& \gamma_{1}\left(14.5-e_{21}\right)=0 \\
& \gamma_{2}\left(6-e_{22}\right)=0 \\
& \gamma_{3}\left(7-e_{23}\right)=0, \gamma_{1}, \gamma_{2}, \gamma_{3} \geq 0
\end{aligned}
$$

We have $J_{1} \subseteq\{1,2,3\}$. For $J_{1}=\varnothing, \gamma_{1}, \gamma_{2}, \gamma_{3}=0$. Then,

$S_{J_{1}}(2.5,6,4.5,14.5,14.5,6,7)=\left\{e_{2} \in \mathbb{R}^{3}: 0 \leq e_{21} \leq 14.5,0 \leq e_{22} \leq 6,2.5 \leq e_{23} \leq 7\right\}$.

For $J_{2}=\{1\}, \gamma_{1}>0, \gamma_{2}, \gamma_{3}=0$. Then,

$S_{J_{2}}(2.5,6,4.5,14.5,14.5,6,7)=\left\{e_{2} \in \mathbb{R}^{3}: 0 \leq e_{21} \leq 14.5, e_{22}=6, e_{23}=7\right\}$.

For $J_{3}=\{2\}, \gamma_{2}>0, \gamma_{1}, \gamma_{3}=0$. Then, 
$S_{J_{3}}(2.5,6,4.5,14.5,14.5,6,7)=\left\{e_{2} \in \mathbb{R}^{3}: 0 \leq e_{21} \leq 14.5, e_{22}=6,2.5 \leq e_{23} \leq 7\right\}$.

For $J_{4}=\{3\}, \gamma_{3}>0, \gamma_{1}, \gamma_{2}=0$. Then,

$S_{J_{4}}(2.5,6,4.5,14.5,14.5,6,7)=\left\{e_{2} \in \mathbb{R}^{3}: e_{21}=14.5, e_{22}=6,2.5 \leq e_{23} \leq 7\right\}$.

For $J_{5}=\{1,2\}, \gamma_{1}, \gamma_{2}>0, \gamma_{3}=0$. Then,

$S_{J_{5}}(2.5,6,4.5,14.5,14.5,6,7)=\left\{e_{2} \in \mathbb{R}^{3}: 0 \leq e_{21} \leq 14.5,0 \leq e_{22} \leq 6, e_{23}=7\right\}$.

For $J_{6}=\{2,3\}, \gamma_{2}, \gamma_{3}>0, \gamma_{1}=0$. Then,

$S_{J_{6}}(2.5,6,4.5,14.5,14.5,6,7)=\left\{e_{2} \in \mathbb{R}^{3}: e_{21}=14.5,0 \leq e_{22} \leq 6,2.5 \leq e_{23} \leq 7\right\}$.

For $J_{7}=\{1,3\}, \gamma_{1}, \gamma_{3}>0, \gamma_{2}=0$. Then,

$S_{J_{7}}(2.5,6,4.5,14.5,14.5,6,7)=\left\{e_{2} \in \mathbb{R}^{3}: 0 \leq e_{21} \leq 14.5, e_{22}=6,2.5 \leq e_{23} \leq 7\right\}$,

$S(2.5,6,4.5,14.5,14.5,6,7)=\bigcup_{q=1}^{7} S_{J_{q}}(2.5,6,4.5,14.5,14.5,6,7)$.

\section{Concluding remarks}

In this paper, a transportation problem in which the costs, supplies and demands represented by heptagonal fuzzy numbers has been studied. A goal programming approach is applied to solve the problem after converting it into the corresponding crisp problem. The stability set of the first kind corresponding to the obtained solution is determined. The advantage of this approach that it is more flexible, can absorb the nature and intricacies of the fuzzy parameters, proves applicable and makes a situation realistic.

\section{Acknowledgements}

The author would like to thank the referees for their suggestive and helpful comments that have led to the improved version of the paper.

\section{References}

[1] Ahmed M.M., Khan A.M., Ahmed F., Sharif UdDin M.D., Incessant allocation method for solving transportation problem, Am. J. Oper. Res., 2016 (6), 236-244.

[2] Bellman R., ZAdeh L., Decision making in a fuzzy environment, Manage. Sci., 1970, 17, 141-164.

[3] ChandRaseKaran S., KoKILA G., SAJu J., Fuzzy transportation problem of hexagon number with $\alpha$-cut and ranking technique, Int. J. Sci. Eng. Appl. Sci., 2015 (1), 530-538. 
[4] ChHibBer P., Bisht D.C.S., SRIVASTAVA D.K., Ranking approach based on incenter in triangle of centroids to solve type-1 and type-2 fuzzy transportation problems, AIP Conference Proc., 2019 (1), 2061.

[5] Dubois D., Prade H., Fuzzy Sets and Systems. Theory and Application, Academic Press, New York 1980.

[6] Gani A.N., ABbas S., A new method for solving intuitionistic fuzzy transportation problem, Appl. Math. Sci., 2013, 7 (28), 1357-1365.

[7] JAIKUMAR K., New approach to solve fully fuzzy transportation problem, Int. J. Math. Appl., 2016, 4 (2-B), 155-162.

[8] KAUR A., KUMAR A., A new method for solving fuzzy transportation problems using ranking function, Appl. Math. Model., 2011, 35 (12), 5652-5661.

[9] MAHESWARI P., VIJAYA M., On initial basic feasible solution (IBFS) of fuzzy transportation problem based on ranking of fuzzy numbers using centroid of incenters, Int. J. Appl. Eng. Res., 2019, 14 (4), $155-164$

[10] MALINI S.U., KENNEDY F.C., An approach for solving fuzzy transportation problem using octagonal fuzzy numbers, Appl. Math. Sci., 2013, 7 (54), 2661-2673.

[11] MATHUR N., SRIVASTAVA P.K., A pioneer optimization approach for hexagonal fuzzy transportation problem, AIP Conference Proc., 2019, (2061), 020030.

[12] Melita Vinoliah E., Ganesan K., Solution of fuzzy transportation problem. A new approach, J. Pure Appl. Math., 2017, 113 (13), 20-29.

[13] NARAYANAMOORTHY S., SARANYA S., MAHESWARI S., A method for solving fuzzy transportation problem (FTP) using fuzzy Russell's method, Int. J. Intel. Syst. Appl., 2013 (2), 71-75.

[14] Osman M.S.A., Qualitative analysis of basic notions in parametric convex programming. I. Parameters in the constraints, Appl. Math., 1977 (22), 318-322.

[15] OSMAn M.S.A., EL- BANNA A.Z.H., Stability of multiobjective nonlinear programming problems with fuzzy parameters, Math. Comp. Sim., 1993 (35), 321-326.

[16] Pathade P.A., Ghadle K.P., Optimal solution of balanced and unbalanced fuzzy transportation problem by using octagonal fuzzy numbers, Int. J. Pure Appl. Math., 2018, 119 (4), 617-625.

[17] Rajarajeswari P., SAHAYA SudHa A., KARTHIKA R., A new operation on hexagonal fuzzy numbers, Int. J. Fuzzy Logic Syst., 2013 (3), 15-26.

[18] RATHI K., BALAMOHAN S., Representation and ranking of fuzzy numbers with heptagonal membership function value and ambiguity index, Appl. Math. Sci., 2014 (8), 4309-4321.

[19] RAMESH KUMAR M., SUBRAMANIAN S., Solution of fuzzy transportation problems with trapezoidal fuzzy numbers using robust ranking methodology, Int. J. Pure Appl. Math., 2018, 119 (16), 3763-3775.

[20] SAHAYA SUdHA A., KARUNAMBIGAI S., Solving a transportation problem using heptagonal fuzzy numbers, Int. J. Adv. Res. Sci., Eng. Techn., 2017, 4 (1), 3118-3125.

[21] Senthilkumar P., Vengataasalam S., A note on the solution of fuzzy transportation problem using fuzzy linear system, J. Fuzzy Set Val. Anal., 2013 (2013), article ID jfsva-00138.

[22] TANAKA H., TCHiHASHI H., Asal K., A formulation of fuzzy linear programming problem based on comparison of fuzzy number, Control Cyber., 1984, 13 (3), 184-194.

[23] ZADEH L.A., Fuzzy sets, Inf. Control, 1965, (8), 338-353. 